\title{
Construcción de ecosistemas de aprendizaje en clases de consulta en formación superior
}

\author{
Construction of learning ecosystems related consultation classes in higher education
}

\author{
Emiliano Foresto ${ }^{1}$ \\ Agustina María Manavella² \\ Rocío Belén Martín
}

\section{Resumen}

En esta investigación se han descrito los rasgos que caracterizan a las clases de consulta universitarias e institutos terciarios. Si bien dichos espacios no se encuentran dentro del currículum prescripto, sí figuran en los mensajes que los docentes fijan en las carteleras. La participación en las clases de consulta es un tanto arbitraria: los días, horarios y espacios en que se ofrecen son fijados por cada docente. La metodología fue un estudio de caso múltiple. Participaron siete docentes pertenecientes a diversas universidades e institutos terciarios de Río Cuarto, Sampacho y Villa Mercedes y diez estudiantes que asistieron a las clases de consulta. Los datos fueron recabados a través de observaciones no participantes y entrevistas individuales y semi-estructuradas. Los resultados muestran la relevancia en la construcción colaborativa de habilidades profesionales y socio-emocionales, la autonomía de trabajo, el aprendizaje a partir de problemas genuinos, la construcción de procesos identitarios y de metas compartidas. Estos rasgos, sumados a las particularidades de los métodos de enseñanza, el lugar en el que se llevan a cabo las clases de consulta y los tiempos de duración, permiten pensar en la complejidad que atraviesan estos ámbitos de aprendizaje.

Palabras clave: clases de consulta; contexto de aprendizaje; enseñanza superior; aprendizaje; ecosistemas de aprendizaje.

\section{Abstract}

In this research, the features that characterize the university consultation classes and tertiary institutes have been described. Although these spaces are not within the prescribed curriculum, they do appear in the messages that teachers post on the billboards. Participation in consultation classes is somewhat arbitrary: the days, times and spaces in which they are offered are set by each teacher. The methodology was a multiple case study. Seven teachers from various universities and tertiary institutes of Río Cuarto, Sampacho and Villa Mercedes participated, as well as ten students who attended the consultation classes. The data were collected through non-participant observations and individual and semi-structured interviews. The results show the relevance in the collaborative construction of professional and socio-emotional skills, work autonomy, learning from genuine problems, the construction of identity processes and shared goals. These features, added to the particularities of the teaching methods, the place where the consultation classes are held and the length of time, allow us to think about the complexity that these learning areas go through.

Key Words: consultation classes; learning context; higher education; learning; learning ecosystems.

\footnotetext{
1 Ingeniero Agrónomo. Docente de Botánica Sistemática Agrícola y Becario Doctoral en CONICET. Universidad Nacional de Río Cuarto (UNRC), Río Cuarto, Córdoba, Argentina. eforesto@ayv.unrc.edu.ar Orcid: https://orcid.org/0000-0002-8196-3030

2 Licenciada en Psicopedagogía. Docente del Taller de Apoyo al Trabajo Final de Grado y Becaria Doctoral en CONICET. Universidad Nacional de Villa María. agustinamanavella@unvm.edu.ar Orcid: https://orcid.org/0000-0002-9159-8559

3 Doctora en Psicología por la Universidad Nacional de San Luis. Profesora Adjunta en el Departamento de Enseñanza de la Ciencia y la Tecnología de la Facultad de Ciencias Exactas, Físicas y Naturales de la Universidad Nacional de Córdoba. rbmartin@unc.edu.ar Orcid: https://orcid.org/0000-0003-3172-0070
}

Recibido: 28/10/2020 - Aprobado: 15/11/2020 


\section{Introducción}

La psicología educacional presenta un marcado interés por estudiar cómo los estudiantes aprenden a partir de su participación e implicación, en colaboración con otros estudiantes, el profesor y otros adultos, en procesos auténticos de investigación y construcción colectiva del conocimiento, en diversos contextos de aprendizaje (Onrubia, 2004). Muchos autores, a lo largo de las últimas décadas, han investigado sobre los rasgos y características de los diversos contextos de aprendizaje -formales, no formales e informales-, pero observamos que son casi nulas las investigaciones que han prestado atención al papel de las "clases de consulta" como un contexto de aprendizaje.

El artículo describe los rasgos que caracterizaron a siete clases de consulta ofrecidas en formación superior, en universidades e institutos terciarios de diferentes ciudades de Argentina, de marzo a diciembre de 2019. La investigación es de carácter exploratorio con un enfoque cualitativo por medio de un estudio de caso múltiple. El estudio brinda un primer acercamiento al conocimiento de la compleja dinámica que se vivencia en estos ámbitos de aprendizaje, y a su vez, pretende alentar a los investigadores y docentes a profundizar en el entendimiento de la circulación y producción de saberes que se producen en contextos de diferente naturaleza, resignificando la idea de poder aprender más allá del aula.

El escrito se organiza en cuatro secciones. En primer lugar, se realiza una revisión bibliográfica haciendo un recorrido por los escritos que se atendieron en la problemática. En segundo lugar, se describen los aspectos metodológicos del trabajo. En tercer lugar, se muestran y discuten los principales resultados alcanzados. Por último, se exponen algunas consideraciones finales sobre los procesos de aprendizaje en el contexto de las clases de consulta en Educación Superior.

\section{Revisión de literatura}

Particularmente, en lo que refiere a las clases de consulta, se ha tenido acceso a los trabajos de Quiroz (1992) y Ruiz-Danegger (1999). Estos autores plantean que los estudiantes denominan a las clases de consulta que se encuentran inmersas dentro de los contextos académicos superiores -universitarios y terciarios-, como "horarios de consulta". En estas clases opcionales los docentes orientan y estimulan la autopreparación de los estudiantes en sus contenidos. Dichos espacios son ofrecidos por la institución, aunque si bien, no se los encuentra dentro del currículum prescripto establecido en los documentos curriculares oficiales, sí figuran en los mensajes que los docentes fijan en las carteleras físicas o en los muros de las plataformas virtuales que utilizan las cátedras para hacer sus comunicados. La participación en dichos ámbitos es un tanto arbitraria: los días, horarios y espacios en que se ofrecen son fijados por cada cátedra e incluso por cada docente en particular (Quiroz, 1992; Ruiz-Danegger, 1999).

En la Psicología Educacional, los estudios sobre los contextos de aprendizaje recibieron una atención creciente en los últimos años (Rinaudo, 2014). Estos contextos nos invitan a pensar el aprendizaje en relación al ámbito o al espacio en el cual se llevan a cabo (Foresto, 2020), como así también a las interacciones que allí acontecen y a los recursos materiales y simbólicos que se utilizan y requieren para la construcción de conocimientos. Recuperando los aportes de Trilla et al., (2003) y Martín (2019), se plantea que, si bien las clases de consulta se ofrecen en los centros de formación universitarios y terciarios, dichas instancias de aprendizaje no se constituyen en contextos educativos formales, dado que estos espacios carecen de la estructuración, organización, obligación y reconocimiento que distinguen a los contextos formales. Por otra parte, si bien las clases de consulta tienen ciertas características vinculadas a los contextos de aprendizaje no formales -tales como: flexibilidad y funcionalidad en cuanto a métodos de enseñanza empleados y a los horarios pautados, atención a las necesidades y demandas de los participantes-, no pueden considerarse a dichos ámbitos como contextos no formales, dado que las clases de consulta se llevan a cabo en el marco institucional del sistema educativo. Al mismo tiempo, dada la intencionalidad educativa que persiguen las clases de consulta, tampoco pueden considerarse como contextos de aprendizaje informales. 
Las particularidades que adquieren las clases de consulta, posibilita pensarlas como espacios donde no hay una clara delimitación que permita identificarlas con un contexto de aprendizaje en particular. En este sentido, en las clases de consulta se producen intromisiones mutuas entre los contextos formales, no formales e informales, funcionando dichos espacios como verdaderos ecosistemas de aprendizaje. El concepto de ecologías de aprendizaje hibrida las potencialidades y características de los variados contextos para crear un entorno promisorio en el que, la construcción de conocimientos posibilite a cada estudiante mantener una estrecha vinculación con el entorno (Martinenco, Martín y García, 2019). Estos ámbitos son verdaderos ecosistemas de aprendizaje que invitan a re-pensar la construcción de conocimientos y habilidades desde una visión más holística, desde factores, dimensiones y una dinámica que facilitan la estimulación de la curiosidad, la indagación, el diálogo, el trabajo colaborativo, los intereses, ideas, pasiones y necesidades, desplazando el centro de gravedad del proceso del sistema docente-escolar al estudiante (Foresto, 2020).

\section{Materiales y métodos}

Se caracterizaron las clases de consulta en ámbitos de formación superior en este estudio de caso múltiple (Stake, 1998). El estudio de caso cualitativo valora las múltiples perspectivas de los interesados, la observación en circunstancias que se producen de forma natural y la interpretación en contexto, posibilitando el estudio de las interacciones entre los participantes en un contexto particular (Simons, 2011).

Cada caso estuvo conformado por el profesor que ofreció la clase de consulta y los estudiantes que participaron de dicha instancia. A los fines de este estudio, se consideraron siete clases de consulta, ofrecidas en los siguientes espacios de formación: Obstetricia, Ingeniería Agronómica, Medicina Veterinaria, Ciencias Biológicas, Diseño Gráfico, Licenciatura en Ciencias Políticas y Profesorado de Educación Inicial. Las clases de consulta mencionadas se llevaron a cabo a lo largo entre marzo-diciembre del 2019, en universidades e institutos terciarios de las localidades de Río Cuarto, Sampacho y Villa Mercedes (Argentina).

Para la recolección de datos se procedió a realizar observaciones no participantes y registro de los intercambios. También se llevaron a cabo entrevistas individuales y semi-estructuradas a profesores y a estudiantes que participaron de dichas instancias. En el próximo apartado referiremos a los profesores a cargo de las clases de consulta estudiadas, con la sigla "P". Para identificar a los estudiantes, utilizaremos la sigla "E".

\section{Resultados y discusión}

Los resultados se organizaron en torno a las siguientes dimensiones: enseñanza en función de intereses y necesidades de los estudiantes; particularidades del contexto y construcción de habilidades.

\section{Enseñanza en función de intereses y necesidades de los estudiantes}

A partir del análisis de datos advertimos que, si bien en estos contextos se retoman los contenidos abordados en las horas cátedra, las propuestas de enseñanza no suelen estar predeterminadas por el profesor, sino que se construyen en el momento, particularmente, en función de los intereses, dudas y necesidades de los estudiantes que asisten y participan de estas instancias:

P: ¡Buen día alumnos! Vamos a comenzar con la clase de consulta... no sé, ¿tienen alguna duda? ¿Quieren que vayamos repasando todas las unidades y vamos viendo un poco todo en las 2 horas que tenemos?

E: Yo profe, si no le molesta le quiero hacer una consulta puntual, que fue por la que vine y después me voy a estudiar a mi casa. A mí me sirve más de esa forma ... Quiero saber: ¿cuántos óvulos por escama hay en la familia Araucariáceas?

P: Sí, ¡no hay problema! Es un solo óvulo por escama y recordá que los conos son muy grandes (prolongando la pronunciación del muy por unos segundos, para hacer referencia a esa particularidad). 
E: ¡Perfecto Profe! Muchas Gracias. (El estudiante saluda y se retira). [Observación de clase de consulta de Ingeniería Agronómica].

Como advertimos anteriormente, el relato recuperado permite reconocer que el desarrollo de las clases en estos contextos adquiere cierta flexibilidad y se organiza en función de las preguntas y de las temáticas que los estudiantes consideran necesario continuar profundizando. Así los estudiantes construyen y adquieren mayor autonomía y autorregulación en sus procesos de aprendizaje.

\section{Particularidades del contexto}

A diferencia de las horas cátedra que se ofrecen en las instituciones de formación superior y se caracterizan por la estructuración en cuanto a la presencialidad, la distribución, el agrupamiento de los estudiantes, la organización de los tiempos y espacios en los que acontecen las clases (Trilla et al., 2003), en las clases de consulta advertimos cierta flexibilidad en los aspectos anteriormente mencionados. Seguidamente se describirán y analizarán dichas particularidades, atendiendo puntualmente al tiempo en el que se llevan a cabo las clases de consulta, a los espacios destinados para dichas instancias y a los métodos de enseñanza.

El tiempo en el que se llevan a cabo las clases de consulta es muy variado y se organiza y establece en común acuerdo, entre el profesor y los estudiantes. En algunas ocasiones, se acuerda un horario de duración y se asiste a un determinado lugar para acceder a dichas clases. En otras instancias, las consultas se llevan a cabo sin un contacto presencial, sino a partir de interacciones en redes sociales. En este tipo de consultas, no se pautan ni fijan de antemano días, horarios ni momentos para plantear las dudas y responder a ellas:

A mí me consultan mucho por WhatsApp, no quieren perder el tiempo. Ya directamente no fijo un horario de consulta, porque me encontraba todos los lunes solo y no iba nadie. En cambio, ellos me mandan WhatsApp de lo que necesitan y yo les respondo y pueden seguir adelante, ya que se destacan por ser muy autónomos. [Entrevista a Profesor de la Licenciatura en Diseño Gráfico.

La flexibilidad que caracteriza a estos contextos les permite a los estudiantes construir mayor autonomía en sus procesos de aprendizaje. Mediante las interacciones con el profesor a través de WhatsApp, los estudiantes autorregulan sus aprendices, evaluando los conocimientos construidos en relación a cada contenido abordado en la cátedra, reconociendo qué temáticas necesitan continuar trabajando y disponiendo del tiempo para generar instancias de diálogo y de interacción con los profesores que acceden a esta modalidad de consultas.

La flexibilización en relación a los diversos modos de interacción para realizar las consultas, no solamente depende de los horarios disponibles y de la autonomía construida en estos contextos de aprendizaje, sino que también cobran relevancia los tipos de consultas a realizar y los elementos que se requieren para aclarar dichas dudas:

(...) También recibía muchas consultas por mail. Había ofrecido este medio dado que muchas veces, para corroborar un dibujo, saber una bibliografía u otro planteo simple, les evitaba tener que ir al campus (...). [Entrevista a Profesora de Ingeniería Agronómica].

La flexibilidad se reconoce también en las conversaciones que se generan entre los profesores y los estudiantes, dado que en estos contextos no solamente se abordan contenidos teóricos y prácticos vinculados a la cátedra, sino que, además se conversa sobre cuestiones de índole personal, haciendo de este ámbito un espacio más personalizado, significativo y ameno, que promueve una relación de cercanía entre profesores y estudiantes. Notamos aquí que los tiempos destinados a las clases de consulta y los horarios en que las mismas se desarrollan son flexibles y se adaptan a las disponibilidades de quienes participan de estos contextos. 
Respecto a los espacios en donde acontecen las clases de consulta, advertimos que también son flexibles y se adaptan a las necesidades de los estudiantes y a los contenidos a abordar:

La dinámica de la clase de consulta es variada, dependiendo de los grupos, del grado de participación que manifiesten. En la asignatura de la cual formé parte durante 25 años aproximadamente se daban modalidades interesantes (...) yo siempre hacía dibujos explicativos y cuadros sinópticos, si era a campo, usaba la "maderita" como pizarrón (...) [Entrevista a Profesor de Ingeniería Agronómica].

La expresión recuperada permite reconocer que, en función de los contenidos a ser trabajados en el espacio de las clases de consulta, las mismas pueden desarrollarse en diversos ámbitos: ya sean aulas, oficinas o en el campo. En este sentido, los elementos, herramientas y recursos implementados para el desarrollo de las clases de consulta varían en función de los temas a abordar y del espacio en el que se ofrezca dicha instancia de enseñanza y aprendizaje. En muchas ocasiones, se habilitan espacios que no estaban destinados para clases de consulta, para tal fin, como suele suceder en las instancias de prácticas supervisadas por los docentes o en las guardias en clínicas; lo cual da cuenta de la flexibilidad que caracteriza a las clases de consulta como contextos de aprendizaje:

Generalmente, el estar 12 horas de guardia con los estudiantes favorece el vínculo docente/ alumno y se generan espacios para evacuar dudas que son no tan convencionales. Luego de controlar un trabajo de parto, realizar el parto, atender consultorio de admisión, atención del piso del Servicio de Materno, realizamos autocríticas. [Entrevista a Profesor de la Licenciatura en Obstetricia].

A veces los espacios donde se desarrollan las consultas no son estancos y pueden ir cambiando de acuerdo a las necesidades de los estudiantes y los contenidos a abordar. El relato de esta docente nos refleja cómo en su materia, evacuar las dudas y realizar las consultas en el hospital in situ, en los espacios habilitados para las guardias, es más valioso que hacerlo en otro lugar, ya que los estudiantes pueden recibir una devolución y construir intercambios con sus profesores en función de las prácticas realizadas, de su desempeño profesional y evaluar su rendimiento y los conocimientos construidos.

La metodología de enseñanza implementada en estos contextos es flexible y se modifica y adapta en función de los contenidos que se estén abordando, de las necesidades de los estudiantes y de los tiempos y espacios en los que las mismas se desarrollan:

Con mi grupo de prácticas, mis pichonas, el día previo al examen final, empecé a interactuar con ellas y me empezaron a mandar mensajes de audio a un grupo que tenemos de WhatsApp para despejar las dudas. Ya era tarde, pero interactuamos un buen rato, yo les hice una breve reseña de las inquietudes que tenían y lo más lindo de todo y gratificante fue que al otro día a todas les fue bien y no sólo porque habían aprobado, sino que el examen, que tiene un formato de preguntas no estructuradas, daba hecho de que habían comprendido y se habían podido apropiar del conocimiento, eso estuvo bien para mí, fue gratificante. [Entrevista a Profesora de la Licenciatura en Obstetricia].

A partir del fragmento de esta entrevista identificamos que la metodología implementada por la profesora para abordar las dudas de las estudiantes se adaptó a la red social en la cual interactuaron, como así también a las particularidades de las dudas y de los contenidos que próximamente iban a ser evaluados. Por otra parte, advertimos la relación cercana y estrecha que se genera entre profesores y estudiantes en estos contextos de aprendizaje, lo cual se pone de manifiesto en el apodo bajo el cual la profesora nombra a sus alumnas, en los horarios en que interactúan y en la creación de un grupo. La predisposición y el compromiso que la profesora construyó con estas alumnas en particular, se evidencia en la elaboración de la reseña en función de las dudas del grupo, como así también en la alegría y satisfacción personal que experimentó tras el desempeño de las estudiantes en la situación de examen. En función del relato de entrevista citado y de 
las observaciones realizadas en estos contextos, reconocemos que en las clases de consulta no existe una metodología específica: los contenidos, el espacio, el tiempo de duración, la predisposición de los profesores y la participación de los estudiantes intervienen a su manera para generar nuevas situaciones de aprendizaje.

\section{Construcción de habilidades}

En el presente apartado haremos mención a las habilidades profesionales y socio-emocionales que se construyen socialmente en el contexto de las clases de consulta, a partir del trabajo y aprendizaje colaborativo y de las interacciones que allí acontecen.

Las habilidades profesionales son aquellas que se circunscriben al contenido técnico y/o práctico de un determinado trabajo o profesión. Dichas habilidades se construyen mediante la experiencia y la fusión de la teoría con la práctica (González Maura, 2006). En el contexto de las clases de consulta, los profesores reconocen las necesidades y demandas de los estudiantes y, en función de las mismas, adaptan los métodos de enseñanza, de manera tal que la forma de abordar estas instancias de aprendizaje, les permita a los participantes construir habilidades vinculadas a la profesión en la que se están formando:

Los alumnos que asisten a clase de consulta, en mi caso que estoy en la cátedra de Prácticas Hospitalarias IV de la Licenciatura en Obstetricia, son los que necesitan un fortalecimiento (...). Para adquirir habilidades que están relacionadas con las prácticas en el hospital para resolver casos clínicos. Todo necesitan que los ayudemos a poder articular teoría con práctica, entonces los métodos que utilizamos apuntan a este propósito. [Entrevista a Profesor de la Licenciatura en Obstetricia].

Las habilidades que se trabajan en las clases de consulta están vinculadas a la articulación entre la teoría y la práctica. La construcción de dichas habilidades promovería que los estudiantes cuenten con las herramientas, recursos y conocimientos necesarios para poder resolver los diversos casos clínicos que se les presenten, en primera instancia, en sus prácticas hospitalarias y, en un futuro, en el ejercicio de su profesión.

Las habilidades profesionales se construyen a partir del trabajo con problemas genuinos, plausibles de presentarse en la realidad, propios de cada profesión. Algunas observaciones y entrevistas realizadas a estudiantes y docentes en el contexto de las clases de consulta, dan cuenta de lo expresado:

E: Profe, tengo dudas con la familia cucurbitáceas, ¿entrará en el parcial?

P: (Agarra el mate y lo golpea) ¿Qué es esto?

E: (Todos responden en coro): ¡un mate profe!

P: ¿Y de dónde sale el mate? ¿Saben que el mate es un zapallo?, se le llama vulgarmente porongo y su nombre científico es Lagenaria siceraria y pertenece a la familia cucurbitáceas (...) continúa ampliando con características botánicas. [Observación de clase de consulta de Ingeniería Agronómica].

La profesora podría haber optado por desarrollar la teoría del concepto explicando minuciosamente cada uno de los caracteres botánicos, pero optó por explicar y desarrollar la teoría a partir de un ejemplo cotidiano, con el que todos los estudiantes están familiarizados. Retomando los estudios de Paoloni (2010), reconocemos que dicha instancia de aprendizaje se constituyó en significativa para los estudiantes que participaron de la clase de consulta, dado que la profesora adaptó la explicación de la temática en función del interés y de las dudas de los participantes, promoviendo que los estudiantes lograran situar la teoría en el contexto de lo que ya saben, de lo que es conocido por ellos. 
En el contexto de una clase de consulta en Medicina Veterinaria, uno de los estudiantes entrevistados también reconoció la importancia del trabajo a partir de problemas genuinos, que impliquen establecer relaciones entre el contexto cotidiano y los conocimientos profesionales:

En una clase de consulta de Producción de Carne, donde veíamos todos lo referido a la bosta de las vacas y su importancia, el profe cuando termina la explicación y aclaramos todas las dudas no dice: una cosa más: "no es bosta, es plata" ... Nunca más me voy a olvidar de la importancia del análisis de la materia fecal en un sistema de producción de feedlot. [Entrevista a estudiante de Medicina Veterinaria].

Advertimos cómo los estudiantes le otorgan significatividad a las clases de consulta donde se vinculan los contextos de enseñanza con diversos aspectos de la vida cotidiana, atendiendo a las inquietudes genuinas que ellos presentan. La valoración que los estudiantes realizan sobre estas instancias está vinculada al reconocimiento de la utilidad que tienen las actividades y las temáticas abordadas en las clases de consulta, para sus aprendizajes profesionales.

En estos contextos, las habilidades profesionales no se construyen de manera aislada, sino a partir de aprendizajes colaborativos. En este sentido, Lave y Wenger (1991) conciben al aprendizaje como un proceso de construcción social, en donde las personas aprenden en contextos reales, a partir de la interacción con otros aprendices y con expertos. Las clases de consulta como contextos de aprendizaje que promueven la colaboración entre pares, estimulan que los estudiantes compartan y discutan ideas, comentarios, puntos de vista, reflexiones y modalidades de resolver diversas situaciones. Seguidamente, recuperamos un fragmento de observación que permite reconocer las particularidades que adquiere la construcción de habilidades profesionales a partir del trabajo colaborativo, en el contexto de las clases de consulta:

E: Profe tenemos que sacar la materia sí o sí los cuatro (haciendo referencia a su grupo de estudio), porque se nos cae la regularidad, así que estamos estudiando juntos y así nos vamos apoyado entre todos.

P: Me parece muy bien, es muy beneficioso que estudien juntos porque pueden resolver las guías y a veces lo que uno no sabe el otro sí lo sabe, además es beneficioso (...). ¡Qué bueno que estudien en grupo!

E: ¡Sí profe! Ahora estamos ayudando al Negro (apodo de uno de los miembros del grupo) a que saque Matemática, así los cuatro podemos tirar Química en el último llamado.

P: ¡Vamos! Ánimo, a seguir estudiando y si tienen alguna duda me escriben por mail y lo charlamos, así no tienen que venir hasta el campus.

E: ¡Gracias profe! (En coro). [Observación de clase de Consulta de Medicina Veterinaria].

En el recorte de observación, advertimos que los estudiantes conformaron un grupo de estudio con sus compañeros, construyendo una identidad colectiva, lo cual promueve que compartan no solamente el cursado de las horas cátedras, sino también la participación en las clases de consulta y horarios de preparación y estudio para rendir exámenes. Esta identidad colectiva permanece en el tiempo, atraviesa diversos contextos y les permite a los estudiantes sentirse parte de un grupo y en función de ello, construir metas de aprendizaje compartidas.

Dado que en las clases de consulta participa un grupo reducido de estudiantes, se generan allí instancias de aprendizaje y vínculos cercanos, lo cual promueve la creación de relaciones personales que trascienden la construcción de habilidades vinculadas exclusivamente al aprendizaje de la profesión. Bar-On (1997) plantea que, las habilidades socio-emocionales influyen en la capacidad para afrontar demandas, desafíos y presiones cotidianas. Dentro de las habilidades socio-emocionales, este autor reconoce la empatía, las relaciones interpersonales y la responsabilidad social. 
Ellos llegan a consulta donde el eje central es despejar alguna duda de la materia, pero muchas veces al generarse un vínculo más estrecho con el estudiante, a veces salen problemas familiares, ellos se abren y a veces son momentos donde se hablan de problemas que escapan al contenido de la materia, pero que a ellos les angustia y eso no les permite continuar con la materia. [Entrevista a Profesor de la Licenciatura en Ciencias Biológicas].

El rol de los docentes va cambiando de acuerdo a las demandas y necesidades de los estudiantes. En estos contextos de aprendizaje, la empatía que el docente construye con el grupo permite generar un vínculo que muchas veces no se logra en las clases de cátedra, las cuales suele ser más masivas.

Tenía una estudiante que siempre dudaba de todo lo que realizaba, no se sentía segura, iba a consulta para tener seguridad, siempre se terminaba aconsejando no solo de los temas de la materia sino, de su forma de ser, de cómo se sentía, de sus emociones y del miedo que ella tenía al tener que enfrentarse con sus estudiantes, a no poder reaccionar. Terminábamos alivianando esa presión emocional que le generaba el tener que realizar sus prácticas o en un futuro estar al frente de un grado. [Entrevista a Profesora de Nivel Inicial].

Recuperando los aportes de Martín, Paoloni y Rinaudo (2019), reconocemos que las clases de consulta se constituyen sobre la base de las relaciones interpersonales que allí se gestan, siendo las experiencias emocionales que acontecen en estos espacios -afectos, empatía, estados de ánimo y sentimientos de pertenencia-, uno de los principales aspectos que promueve la participación de estudiantes y profesores en estos contextos de aprendizaje.

\section{Conclusiones}

Este estudio permitió reflexionar acerca de las particularidades que adquieren los procesos educativos en el contexto de las clases de consulta en formación superior. Tomando como referencia las categorías que emergieron del análisis de los datos, advertimos que en las clases de consulta se generan instancias de enseñanza y aprendizaje que promueven la construcción colaborativa de habilidades profesionales y socio-emocionales, la autonomía de trabajo, el aprendizaje a partir de problemas genuinos, la construcción de procesos identitarios y de metas compartidas. Estos contextos de aprendizaje se caracterizan por la flexibilidad que presentan en relación a los métodos de enseñanza empleados, el lugar en el que se llevan a cabo y los tiempos de duración; lo cual depende de las necesidades e intereses de los estudiantes que participan en estas instancias, como así también de las particularidades de los contenidos a abordar.

Las clases de consulta son consideradas como un contexto de aprendizaje alternativo para muchos estudiantes que tienen el propósito de comprender los conceptos, avanzar en su carrera y lograr la regularidad. Dichos contextos surgen de la necesidad de ayuda por parte de los estudiantes, ante determinados contenidos académicos que consideran que necesitan continuar reforzando. Estos contextos de aprendizaje pueden interpretarse como el sostén o andamiaje que necesitan los estudiantes para construir, complementar y complejizar los contenidos enseñados en la educación superior, a partir de la interacción y de la ayuda de otros (Wood, Bruner y Ross, 1976; Manavella y Martin,2017).

A raíz del estudio realizado y de la revisión de literatura sobre el tópico, consideramos de suma relevancia avanzar en el estudio de las particularidades que adquieren los procesos de enseñanza y aprendizaje, en contextos de diferente naturaleza.

\section{Agradecimiento}

Esta publicación obtuvo el financiamiento de: El Fondo de Asistencia Internacional de los Estudiantes y Académicos Noruegos (SAIH). 


\section{Lista de referencia}

Bar-On, R. (1997). EQ-i, Bar-On Emotional Quotient Inventory: A measure of emotional intelligence. (Technical manual). Toronto, Canada: Multi-Health Systems.

Foresto E. (2020). Aprendizajes formales, no formales e informales. Una revisión teórica holística. Contextos de Educación, 29 (21), 24-36. Recuperado de: http://www2.hum.unrc.edu.ar/ojs/index.php/contextos/ article/view/1142/1234

González Maura, V. (2006). La formación de competencias profesionales en la universidad. Reflexiones y experiencias desde una perspectiva educativa. XXI, Revista de Educación, 8, 175-187. Recuperado de: https://core.ac.uk/download/pdf/41563302.pdf

Lave, J. y Wenger, E. (1991). Situated Learning: Legitimate Periperal Participation. Cambridge, UK: Cambridge University Press.

Manavella, A. M., y Martín, R. B. (2017). Motivos y expectativas de la demanda de apoyo pedagógico en educación secundaria. Páginas de Educación, 10 (2), 44-56.

Martín, R. B. (2019). Perspectiva teórica sobre el estudio de los contextos y comunidades de aprendizaje. En R. B. Martín, M. C. Rinaudo y P. V. Paoloni. (Ed.), Comunidades: estudios y experiencias sobre contextos y comunidades de aprendizaje (pp.75-131). Villa María: EDUVIM.

Martín, R.B.; Paoloni, P. V. y Rinaudo, M. C. (2019). Senderos promisorios para futura investigación sobre comunidades de aprendizaje y de práctica. En R. B. Martín, M. C. Rinaudo y P. V. Paoloni (Ed.), Comunidades. Estudios y experiencias sobre contextos y comunidades de aprendizaje (pp. 197-207).

Martinenco, R. B.; Martín, R. B. y García, L. (2019). Ecologías de aprendizaje. Una reflexión sobre el aprendizaje en diversos contextos. En D. J. Michelini, S. Otero y M. I. Crabay (Eds.), Convivir en un mundo con fronteras (pp. 1-7).

Onrubia, J. (2004). Las aulas como comunidades de aprendizaje. Revista Electrónica Trabajadores de la Enseñanza T.E. (249). 14-15. Recuperado de: http://www.fe.ccoo.es/publicaciones/TE/249/249.pdf

Paoloni, P. (2010). Factores personales que inciden en la motivación para el aprendizaje. En M.C. Rinaudo y D. Donolo. (Comp.), Estudios sobre motivación: enfoques, resultados, lineamientos para acciones futuras (pp.35-66). Río Cuarto: Universidad Nacional de Río Cuarto.

Quiroz, R. (1992). Obstáculos para la apropiación del contenido académico en la escuela secundaria. Infancia y Aprendizaje, 55, 45-58.

Rinaudo, M. C. (2014). Estudios sobre los contextos de aprendizaje: arenas y fronteras. En P. V. Paoloni, M. C. Rinaudo y A. González Fernández, A. (Comps.) Cuestiones en Psicología Educacional. Perspectivas teóricas, metodológicas y estudios de campo. (pp. 163-205) Tenerife: Sociedad Latinoamericana de Comunicación Social (SLCS). Recuperado de: http://www.cuadernosartesanos.org/2014/cdeo1.pdf

Ruiz-Danegger, C. (1999, mayo). El currículum universitario desde la perspectiva de los estudiantes: el caso de las" clases de apoyo" en química. Presentado en las IV Jornadas Nacionales y I Internacionales de Enseñanza Universitaria de Química, Universidad Nacional del Litoral. Santa Fe, Argentina.

Simons, H. (2011). El estudio de caso: teoría y práctica: Madrid: Morata.

Stake, J. (1998). Investigación con estudios de caso. Madrid, España: Morata. 
Trilla, J.; Gros, B.; López, F y Martín, M. J. (2003). La educación fuera de la escuela. Ámbitos no formales y Educación Social. Barcelona, España: Ariel Educación.

Wood, D., Bruner, J. S. y Ross, G. (1976). The role of tutoring in problem solving. Journal of Child Psychology and Psychiatry, 17, 89-100. 